VOL. $70(2004) \quad[481-488]$

\title{
ABELIAN GROUPS AS INNER MAPPING GROUPS OF LOOPS
}

\author{
Asif Ali And John Cossey
}

The question of which Abelian groups can be the inner mapping group of a loop has been considered by Niemenmaa, Kepka and others. We give a construction which shows that many finite Abelian groups can be the inner mapping group of a loop.

\section{INTRODUCTION}

Despite the title, this paper is about groups; and all groups in this paper are finite. However the question we consider here has its origin in the theory of loops and our results have applications to loops. We shall be concerned in this paper with finite groups with the following structure. Suppose that $G$ is a group with a subgroup $H$ and transversals $A$ and $B$ for $H$ in $G$ which satisfy $[A, B] \leqslant H$. We say that transversals satisfying this condition are $H$-connected and if $A=B$ we shall say that $A$ is $H$-selfconnected. Groups $G$ with this structure arise naturally as the multiplication groups of loops, with $H$ the inner mapping group of the loop, and have been studied extensively by Niemenmaa and others. We refer the interested reader to $[\mathbf{5 , 6 , 7 , 8 ]}$ in particular for the translation of our results to loops. The objects we study in this paper are 4-tuples $(G, H, A, B)$, where $G$ is a group, $H$ a subgroup of $G$ and $A, B$ transversals for $H$ in $G$. Niemenmaa and his collaborators have been interested in particular in groups satisfying the following hypothesis:

( $\alpha)(G, H, A, B)$ where $H$ is a corefree Abelian subgroup of $G, A$ and $B$ are $H$-connected transversals for $H$ in $G$ and $G=\langle A, B\rangle$.

We shall also say that $G$ satisfies $(\alpha)$ if $G$ has a subgroup $H$ and transversals $A$ and $B$ for $H$ in $G$ with $(G, H, A, B)$ satisfying $(\alpha)$. Only a small number of $(G, H, A, B)$ satisfying $(\alpha)$ are known and the results of Niemenmaa, Kepka and others are mainly negative. Our main aim in this paper will be to give a method for constructing 4-tuples $(G, H, A, B)$ satisfying $(\alpha)$ which shows that many Abelian groups occur as inner mapping groups of loops. We shall also be concerned in this paper with the structure of $G$ and the way $H$ is embedded in $G$ when $H$ is Abelian.

We shall follow the usage of Doerk and Hawkes [1, Definition A4.19] and define a homocyclic Abelian group to be the direct product of cyclic groups of the same prime

Received 6gh July, 2004

Copyright Clearance Centre, Inc. Serial-fee code: 0004-9727/04 \$A2.00+0.00. 
power order and we note that a finite Abelian group can be written as the direct product of homocyclic subgroups, each of different exponent. If $J \cong Z_{p^{n}}^{k}$, with $Z_{p^{n}}$ cyclic of order $p^{n}$, we say $J$ is homocyclic of exponent $p^{n}$ and rank $k$. If $J$ is an Abelian group and $J=J_{1} \times \cdots \times J_{n}$ with each $J_{i}$ homocyclic and each $J_{i}$ of different exponent, we call the $J_{i}$ homocyclic components of $J$. The results of Niemenmaa and others indicate that if a homocyclic component of $J$ of maximal $p$-power exponent is cyclic for some prime $p$ then there is no $(G, H, A, B)$ satisfying $(\alpha)$ with $H \cong J$; a number of special cases are dealt with in $[2, \mathbf{3}, \mathbf{4}, \mathbf{5}, \mathbf{6}, \mathbf{7}, \mathbf{8}]$. Our main result is the following.

THEOREM 1. Let $J$ be an Abelian group with no cyclic homocyclic component and no homocyclic component of odd rank and exponent $2^{a}$ with $a>1$. Then there is a 4-tuple $(G, H, A, A)$ satisfying $(\alpha)$ with $F(G)=H \times Z(G)$ and $H \cong J$.

The proof of Theorem 1 is constructive. We shall also look for conditions on $H$ which will ensure that 4 -tuples $(G, H, A, B)$ satisfying the hypothesis $(\alpha)$ will exist. Our aim will be to consider the structure of 4 -tuples $(G, H, A, B)$ which satisfy $(\alpha)$ but are minimal in the sense that if $1 \neq N$ is normal in $G$ then the 4-tuple given by the quotient $G / N$ does not satisfy $(\alpha)$. Note that the properties of $H$ being Abelian and the transversals $A$ and $B$ being $H$-connected and generating $G$ are preserved under quotients; however $H$ being corefree may not be preserved.

Theorem 2. Suppose that $(G, H, A, B)$ satisfies $(\alpha)$. Suppose further that in any quotient of $G$ the image of $H$ is not corefree. Then

(i) $F(G)$ is a $p$-group and $H$ is an elementary Abelian $p$-group for some prime $p$;

(ii) $Z(G)$ is cyclic and $\sigma(G)$ is the normal closure of a single element;

(iii) if $F(G)$ is Abelian, then $F(G)=H \times Z(G)$ and $\sigma(G)=H \times \sigma(Z(G))$.

Here $\sigma(G)$ denotes the socle of $G$, the subgroup generated by all minimal normal subgroups of $G$. Note that if $F(G)$ is Abelian, then $H \times \sigma(Z(G))$ is the normal closure of a single element. An open question is whether this condition is also sufficient: if $G$ is a group with $F(G)$ an elementary Abelian $p$-group for some prime $p, G / F(G)$ Abelian, $Z(G) \neq 1$ and $F(G)$ the normal closure of a single element, can we find $H \leqslant F(G)$ and transversals $A, B$ for $H$ in $G$ such that $(G, H, A, B)$ satisfies $(\alpha)$. We know of no counterexample. In some special cases we were able to show that this condition is sufficient. The ideas involved can be generalised and we used them to produce the examples in Theorem 1.

As might be expected, in attempting to prove Theorem 1 without any restriction on the order of $J$, the primes 2 and to a lesser extent 3 cause problems. For these primes the general procedures we use to construct examples break down. For the prime 3 we are able to construct examples using ideas similar to those for the general 
case. We are grateful to Mike Newman for some useful discussions and some computer calculations which lead to the specific examples for $H$ a homocyclic $p$-group of rank 2 for $p=3$. The computer calculations were for the case of an elementary Abelian group $H$ of order 9 and they indicate that such examples may be more common than expected; for the group $G$ of order 108 with $H$ of order 9 constructed, the computer search found 18 self-connected transversals $A$ which gave a 4 -tuple satisfying $(\alpha)$. (It is worth observing that since the 9 non-central transversals can be chosen arbitrarily there are $9^{9}=3^{18}$ possible transversals, so it is quite rare for a transversal to give a 4 -tuple satisfying $(\alpha)$.) For $p=2$ we can use our ideas to reduce the case of a general homocyclic 2-group to constructing examples for $H$ a homocyclic 2-group of arbitrary exponent and rank 3 but have only been able to produce examples for exponents 2 and 4. A computer calculation on a group of order $2^{4} 7$ produces examples (again we are grateful to Mike Newman for useful discussions and help with the computer calculations). Further calculations generalise these examples to the exponent 4 case (we do not include the details here), but we have been unable to extend the examples.

In all the examples $(G, H, A, B)$ we construct in this paper, we use $H$-selfconnected transversals $(A=B)$; such examples give rise to commutative loops. We conjecture that in all the cases where we have found $H$-self-connected transversals there will also be connected transversals that are not self-connected. This is supported by some computer experimentation, but we have not established it in general.

\section{Preliminaries}

Before we begin the proofs of our results, we collect together some technical lemmas we shall need. Some of them are well known results which are given here for convenience. Others are presumably also known, but we have not found them in the form we need and so have included proofs. Throughout, $G$ will denote a group with a subgroup $H$ with $H$-connected transversals $A$ and $B$. We shall denote by core $_{G}(H)$ the core of $H$ in $G$ (that is the largest normal subgroup of $G$ contained in $H$ ), by $Z(G)$ the centre of $G$ and by $F(G)$ the Fitting subgroup of $G$ (that is, the largest nilpotent normal subgroup of $G$ ). The first group collects together known results about the structure of such groups.

LEMMA 1.

(i) ([6, Lemma 2.5.)] If core $_{G}(H)=1$, then $N_{G}(H)=H \times Z(G)$.

(ii) ([4, Lemma 2.1.)] If $H$ is Abelian, then $H$ is subnormal in $G$.

(iii) ([7, Theorem 3.4.)] If $H$ is Abelian, then $G$ is soluble.

LEMMA 2.

(i) If $H$ is Abelian, then $H \leqslant F(G)$.

(ii) If $H$ is Abelian, $G / F(G)$ is Abelian. 
Proof: The first claim follows immediately from the fact that $H$ is a nilpotent subnormal subgroup of $G$.

The second claim follows from the following easy calculation. Since $A$ and $B$ are transversals for $H$ in $G$, if $x$ and $y$ are elements of $G$, we can write $x=a u$ and $y=b v$, with $a \in A, b \in B$ and $u, v \in H$. But then

$$
[x, y]=[a u, b v]=[a, b]^{u v}[u, b]^{v}[a, v]^{u}[u, v] .
$$

The result now follows from the fact that $[u, v]=1$ and $[a, b], u, v \in H \leqslant F(G)$.

Note that Lemma 2 depends only on Lemma 1 (i) and (ii) and then Lemma 1 (iii) follows from Lemma 2.

Lemma 3. Suppose that $\left(G_{0}, H_{0}, A_{0}, B_{0}\right)$ and $\left(G_{1}, H_{1}, A_{1}, B_{1}\right)$ are 4 -tuples satisfying $(\alpha)$. Then $\left(G_{0} \times G_{1}, H_{0} \times H_{1}, A_{0} \times A_{1}, B_{0} \times B_{1}\right)$ satisfies $(\alpha)$.

Proof: Set $G=G_{0} \times G_{1}, H=H_{0} \times H_{1}, A=A_{0} \times A_{1}$ and $B=B_{0} \times B_{1}$. Clearly $A$ and $B$ are transversals for $H$ in $G$. To see that they are $H$-connected, just observe that if $a_{0} \in A_{0}, a_{1} \in A_{1}, b_{0} \in B_{0}$ and $b_{1} \in B_{1}$, then $\left[a_{0} a_{1}, b_{0} b_{1}\right]=\left[a_{0}, b_{0}\right]\left[a_{1}, b_{1}\right] \in H$. Since all of $A_{0}, A_{1}, B_{0}, B_{1}$ contain the identity, $A_{0}, A_{1}$ are subsets of $A$ and $B_{0}, B_{1}$ are subsets. of $B$. Thus $\left\langle A_{0}, B_{0}\right\rangle=G_{0} \leqslant\langle A, B\rangle$ and $\left\langle A_{1}, B_{1}\right\rangle=G_{1} \leqslant\langle A, B\rangle$ and so $G=\langle A, B\rangle$. That $H$ is corefree is clear.

The next result is well known and comes in many forms. We do not know a reference for it in the form we want and so give a proof. It will play a crucial role in the proof of Theorem 2 .

Lemma 4. Let $F$ be the field of prime order $p$ and let $G$ be an Abelian $p^{\prime}$-group. Let $M$ be a (finite) $F G$-module of order $p^{n}$ and $V$ a subspace of order $p^{n-1}$. Let $M=M_{1} \oplus \cdots \oplus M_{k}$ with each $M_{i}$ irreducible.

(i) If $M_{i} \cong M_{j}$ for some $i \neq j$, then $V$ contains a non-zero submodule of $M$.

(ii) If the $M_{j}$ are pairwise non-isomorphic and $n \geqslant 2$, then there is a maximal subspace $U$ which contains no non-zero submodule of $M$.

Proof: Note that since $G$ is a $p^{\prime}$-group all $F G$-modules are completely reducible.

(i) Suppose that $L=M_{i} \oplus M_{j} \leqslant M$ with $M_{i} \cong M_{j}$. If $L \leqslant V$ there is nothing to prove, so we may assume that $L \cap V$ has index $p$ in $L$. it is enough to show that $L \cap V$ contains a non-zero submodule of $L$. If $N$ is a submodule of $L$ with $0 \neq N \neq L$ then $N$ must be irreducible and isomorphic to $M_{i}$. Suppose that $\left|M_{i}\right|=p^{t}$. Then $L$ contains $p^{t}$ irreducible submodules and each of these irreducible submodules is contained in $\left(p^{t}-1\right) /(p-1)$ subspaces of order $p^{2 t-1}$. Since $G$ is Abelian there are 
exactly $p^{t}+1$ irreducible submodules of $L$ and no proper subspace of $L$ can contain more than one irreducible submodule, there are $\left(p^{t}+1\right)\left(p^{t}-1\right) /(p-1)$ subspaces of order $p^{2 t-1}$ containing an irreducible submodule. But the number of subspaces of order $p^{2 t-1}$ is $\left(p^{2 t}-1\right) /(p-1)$ and the result follows.

(ii) The proof will be by induction on $n$. Note first that if $M$ is irreducible, then any subspace of order $p^{n-1}$ will do, and so we may assume that $M$ is reducible. Suppose then that $n=2$. Then $M=M_{1} \oplus M_{2}$ wih $M_{1}$ and $M_{2}$ non-isomorphic and each of order $p$. But then if $0 \neq u \in M_{1}$ and $0 \neq v \in M_{2}$, the subspace $\langle u+v\rangle$ has order $p$ and is not a submodule (since $M_{1}$ and $M_{2}$ are the only submodules of $M$ ). Thus the result is true for $n=2$.

Now suppose the result is true for orders less than $p^{n}$ and $M$ has order $p^{n}$, $n>2$. Since $M$ is reducible, we can write $M=M_{1} \oplus M_{2}$ and we may assume that $p^{n}>\left|M_{1}\right|=p^{t} \geqslant p^{2}$. Now choose $N_{1}$ to be a subspace of $M_{1}$ of order $p^{t-1}$ which contains no non-zero submodule of $M_{1}$. Choose $N_{2}$ to be a subspace of $M_{2}$ of order $p^{n-t-1}$ which contains no non-zero submodule of $M_{2}$ (and note that $N_{2}=0$ if $\left|M_{2}\right|=p$ ). Now let $u \in M_{1} \backslash N_{1}$ and $v \in M_{2} \backslash N_{2}$ and set $N=N_{1} \oplus N_{2} \oplus\langle u+v\rangle$. If $N$ contains a non-zero submodule of $M$ we must have either $N \cap M_{1}$ or $N \cap M_{2}$ contains a non-zero submodule. But $N \cap M_{1}=N_{1}$ and $N \cap M_{2}=N_{2}$ and hence $N$ does not contain a non-zero submodule. Also $|N|=p^{t-1} p^{n-t-1} p=p^{n-1}$ and the result follows.

\section{Proofs}

Proof of Theorem 2: Suppose that $(G, H, A, B)$ is a group satisfying $(\alpha)$, so that $H$ is a corefree Abelian subgroup $H, A$ and $B$ are $H$-connected transversals $A$ and $B$ and $\langle A, B\rangle=G$.

Since $N_{G}(H)=H \times Z(G) \leqslant F(G)$ and $H<F(G)$, we have $H<N_{G}(H)$ and then $Z(G) \neq 1$. Let $Z$ be a minimal normal subgroup of $G$ contained in $Z(G)$. Then $|Z|=p$ for some prime $p$.

Now consider $G / Z$ and suppose that $H Z / Z$ is not normal in $G / Z$. Then, if $K / Z$ is the core of $H Z / Z$ in $G / Z$, we have $H^{*}=H K / K$ Abelian, $A^{*}=\{a K: a \in A\}$ and $B^{*}=\{b K: b \in B\},\left[A^{*}, B^{*}\right] \leqslant H^{*}$ and $\left\langle A^{*}, B^{*}\right\rangle=G^{*}=G / K$ and so $G^{*}$ satisfies $(I M G)$, a contradiction. Thus $H Z$ is a normal subgroup of $G$. Since $(H Z)^{p} \leqslant H$ and is normal in $G$ we have $(H Z)^{p}=1$ and so $H$ is an elementary Abelian $p$-group. If $Z(G)$ is not cyclic, then there is a minimal normal subgroup $W \leqslant Z(G)$ with $Z \cap W=1$. By the previous argument we must have $H W$ normal in $G$. Since $H Z \neq H W$ we have $H \leqslant(H Z \cap H W) \neq H Z$ and so we must have $H=H Z \cap H W$. But then $H$ would be normal in $G$, a contradiction. Thus $Z(G)$ is a cyclic $p$-group.

Now suppose that $N$ is a minimal normal subgroup of $G$. Then $N$ normalises $H$, 
since $H$ is subnormal (Robinson [9, 13.3.7]) and so $N \leqslant H \times Z(G)$. It follows that the socle $\sigma(G)$ of $G$ is contained in $H \times Z(G)$. Since $H \times Z(G)$ is a $p$-group so is $\sigma(G)$ and hence $F(G)$ is a $p$-group. But then $G / F(G)$ is an Abelian $p^{\prime}$-group, since if a Sylow $p$-subgroup $P / F(G)$ of $G / F(G)$ were nontrivial, $P$ would be a normal nilpotent subgroup of $G$. Since $\sigma(G)$ is elementary Abelian we have $\sigma(G) \leqslant H \times \sigma(Z(G))$. Then $\sigma(G)=\sigma(G) \cap(H \times Z(G))=(\sigma(G) \cap H) \times Z(G)$ and Lemma 4 tells us that $\sigma(G)$ can not contain two minimal normal subgroups isomorphic as $G$-modules. It follows that, as a $G / F(G)$-module, $\sigma(G)$ is a quotient of the regular module and hence a one generator module. In terms of subgroups, this says that $\sigma(G)$ is the normal closure of a single element.

Now suppose that $F(G)$ is Abelian. Then $F(G) \leqslant N_{G}(H)$ and so by Lemma 1(i) we have $F(G) \leqslant N_{G}(H)=H \times Z(G) \leqslant F(G)$. Then $H \times Z(G)=H \times \sigma(Z(G))=\sigma(G)$, since $H \times Z(G)$ is a normal subgroup with $G / C_{G}(H \times Z(G))$ a $p^{\prime}$-group. This completes the proof of the theorem.

Proof of Theorem 1: We begin by observing that if the theorem is true for all homocyclic components of $J$ it will be true for $J$ by Lemma 3. Hence it is enough to consider $J$ homocyclic (and satisfying the hypotheses of the theorem).

Now consider $G\left(p^{n}, d\right)=X w r Y$ (the standard wreath product), with $X$ cyclic of order $p^{n}$ and $Y$ cyclic of order $d, p$ a prime, $d>2$ not divisible by $p$. Set $G=G\left(p^{n}, d\right)$. Note that the base group of $G$ is its Fitting subgroup and is a $d$ generator homocyclic group of exponent $p^{n}$. Suppose $X=\langle x\rangle, Y=\langle y\rangle$ and $F(G)=\left\langle x_{0}, \ldots, x_{d-1}\right\rangle$, with $x_{i}^{y^{j}}=x_{i+j}$ (where we take $i+j$ modulo $d$ ). If we put $z=x_{0} \ldots x_{d-1}$ then $\langle z\rangle=Z(G)$. Now set $H=\left\langle x_{0}, x_{1}, x_{3}, \ldots, x_{d-1}\right\rangle$ (if $d=3$, take $H=\left\langle x_{0}, x_{1}\right\rangle$ ),

$$
A=B=\left\{z^{i} y^{j}: 0 \leqslant i \leqslant p^{n}-1,0 \leqslant j \leqslant d-1, j \neq 1\right\} \cup\left\{z^{i} y x_{1}: 0 \leqslant i \leqslant p^{n}-1\right\} .
$$

We claim that $(G, H, A, B)$ satisfies $(\alpha)$.

It follows immediately from the fact that $H Z(G) Y=G$ that $A$ is a transversal for $H$ in $G$. To see that $A$ is $H$-self-connected, observe that the only non-trivial commutators in $[A, A]$ are $\left[z^{i} y x_{1}, z^{i} y^{j}\right]=\left[y x_{1}, y^{j}\right]=x_{1}^{-1} x_{j+1}$ for $2 \leqslant j \leqslant d-1$. It now follows that $[A, A] \leqslant H$. Next observe that $z \in A$ and so $Z(G) \leqslant\langle A\rangle$. Since $y^{j}=z^{-1}\left(z y^{j}\right) \in\langle A\rangle$ for all $j \neq 1$ we also have $Y \leqslant\langle A\rangle$. We then get $x_{1} \in\langle A\rangle$ and since $G$ is generated by $Y$ and $x_{1}, G=\langle A\rangle$. Since any normal subgroup of $G$ contained in $F(G)$ must contain an element $x_{0}^{i_{0}} \ldots x_{d-1}^{i_{d-1}}$ with $i_{2} \neq 0 \bmod p^{n}, H$ is corefree. Thus $(G, H, A, B)$ satisfies $(\alpha)$.

We have constructed a 4 -tuple $(G, H, A, B)$ satisfying $(\alpha)$ for $H$ an $f$ generator homocyclic $p$-group with $f+1>2$ and prime to $p$. The next step is to consider the case $f+1$ divisible by $p$. For $p \geqslant 5$ we observe that we can write $f=k p-1$ for some 
integer $k \geqslant 1$ and then $f=(k-1) p+2((p-1) / 2)$. By Lemma $3, G\left(p^{n}, p+1\right)^{k-1}$ $\times G\left(p^{n},(p+1) / 2\right)^{2}$ satisfies $(\alpha)$ with $H$ homocyclic of rank $f$ and exponent $p^{n}$.

For $p=3$, if $f+1$ is divisible by 3 we can write $f=3 k+2$ and since for $=3 k$ $G\left(3^{n}, 3 k+1\right)$ satisfies $(\alpha)$ with $H$ homocyclic of rank $3 k$ and exponent $3^{n}$, it will be enough by Lemma 3 to find $(G, H, A, B)$ satisfying $(\alpha)$ with $H$ homocyclic of rank 2 and exponent $3^{n}$. We construct such a group.

Let $F$ be a homocyclic Abelian group of rank 3 and exponent $3^{n}$, generated by $\{u, v, z\}$. Let $Y=\langle y\rangle$ be a cyclic group of order 4 and define an action of $Y$ on $F$ by $u^{y}=v, v^{y}=u^{-1}, z^{y}=z$. Let $G$ be the semidirect product of $F$ and $Y$ and let $H=\langle v, u z\rangle$. Then $H$ is homocyclic of rank 2 and exponent $3^{n}, Z=\langle z\rangle=Z(G)$ and $H \times Z=F=F(G)$. It is easy to check that $H$ is corefree. We now define a transversal for $H$ in $G$ by $A=B=\left\{z^{i} y^{j} w_{i, j}: 0 \leqslant i \leqslant 3^{n}-1,0 \leqslant j \leqslant 3, w_{i, 0}=w_{i, 3}\right.$ $\left.=1, w_{i, 1}=w_{i, 2}=v u z\right\}$. Since $z$ and $v u z$ are in $A$ we have $u z \in\langle A\rangle$ and it follows easily that $\langle A\rangle=G$. To show that $(G, H, A, A)$ satisfies $(\alpha)$ it will be enough to show that $[A, A] \leqslant H$. We claim it is enough to show that $\left[y^{3}, u v\right] \in H$. To see this, take $z^{i} y^{j} w_{i, j}$ and $z^{k} y^{l} w_{k, l}$ in $A$. Then $\left[z^{i} y^{j} w_{i, j}, z^{k} y^{l} w_{k, l}\right]=\left[y^{j} w_{i, j}, y^{l} w_{k, l}\right]=\left[y^{j}, w_{k, l}\right]\left[w_{i, j}, y^{l}\right]$. If $j$ or $l$ is 0 then both commutators in this product are 1 . If $\{j, l\}=\{1,2\}$ then the product is $\left[y^{2}, v u z\right][v u z, y]=\left[y^{2}, v u\right][v u, y]=\left[y^{2}, v u\right][y, v u]^{-1}$ (or its inverse). But $y^{2}$ inverts elements of $\langle u, v\rangle$ and so $[y, v u]^{-1}=[y, v u]^{y^{2}}$ and hence $[y, v u]^{y^{2}}\left[y^{2}, v u\right]$ $=\left[y^{3}, v u\right]$. If $j=l$, then the commutators are inverses of each other and so their product is 1 . Finally if one of $j, l$ is 3 and the other is either 1 or 2 ; say $j=3$ and $l=1$ or $l=2$. Since $w_{i, 3}=1$ and $w_{k, 1}=w_{k, 2}$ we have $\left[y^{3}, w_{k, 1}\right]=\left[y^{3}, v u z\right]=\left[y^{3}, v u\right] \in H$. Thus in both cases we need to show that $\left[y^{3}, v u\right] \in H$. Now $\left[y^{3}, v u\right]=(v u)^{-y^{3}}(v u)$ $=\left(v^{-1} u\right)^{-1} v u=v^{2} \in H$. Thus $(G, H, A, A)$ satisfies $(\alpha)$.

For $p=2$, if $f+1$ is even and at least 4 (since $f>1$ ) we can write $f=3+2 k$. Again $G\left(2^{n}, 2 k+1\right)$ satisfies $(\alpha)$ with $H$ homocyclic of rank $2 k$ and exponent $2^{n}$ and so it will be enough to find $(G, H, A, B)$ satisfying $(\alpha)$ with $H$ homocyclic of rank 3 and exponent $2^{n}$. We have only been able to do this by computer search for $n=1,2$. We give the details of the group for $n=1$ only.

For $n=1$, let $F$ be elementary Abelian of order 16 with generators $u, v, w, z$ and let $X=\langle x\rangle$ be a cyclic group of order 7. Define an action of $x$ on $F$ by $u^{x}=v$, $v^{x}=w, w^{x}=u v, z^{x}=z$. It is easy to check that $x$ acts as an automorphism of order 7. Let $G$ be the semidirect product of $F$ and $X$ and then set $H=\langle u, v, w z\rangle$, so that $H$ has order 8 and index 14 in $G$. We take as our transversal $A$ the set $\left\{a_{j} z^{i} x^{j}\right.$ : $\left.i=0,1,0 \leqslant j \leqslant 6, a_{j}=1, j=0,1,5, a_{2}=w, a_{3}=v, a_{4}=u, a_{6}=u v w\right\}$. It is clear that $G=\langle A\rangle$ and that $H$ is corefree. To see that $[A, A] \leqslant H$ is a straightforward though tedious calculation which we suppress. 


\section{REFERENCES}

[1] K. Doerk and T.O. Hawkes, Finite soluble groups (de Gruyter, Berlin, New York, 1992).

[2] T. Kepka, 'On the abelian inner permutation groups of loops', Comm. Algebra 26 (1998), 857-861.

[3] T. Kepka and M. Niemenmaa, 'On loops with cyclic inner mapping groups', Arch. Math. 60 (1993), 233-236.

[4] M. Niemenmaa, 'On the structure of the inner mapping groups of loops', Comm. Algebra 24 (1996), 135-142.

[5] M. Niemenmaa, 'On finite loops whose inner mapping groups are abelian', Bull. Austral. Math. Soc. 65 (2002), 477-484.

[6] M. Niemenmaa and T. Kepka, 'On multiplication groups of loops', J Algebra 135 (1990), 112-122.

[7] M. Niemenmaa and T. Kepka, 'On connected transversals to abelian subgroups in finite groups', Bull. London Math. Soc. 24 (1992), 343-346.

[8] M. Niemanmaa and T. Kepka, 'On connected transversals to abelian subgroups', Bull. Austral Math. Soc. 49 (1994), 121-128.

[9] D.J.S. Robinson, $A$ course in the theory of groups, Graduate Texts in Mathematics 80 (Springer-Verlag, New York; 1982).

Department of Mathematics

Quaid-E-Azam University

Islamabad

Pakistan
Mathematics Department Mathematical Sciences Institute Australian National University Canberra, ACT 0200

Australia 